Title

Posttraumatic growth and its measurement: A closer look at the PTGI's psychometric properties and structure

\author{
Author list

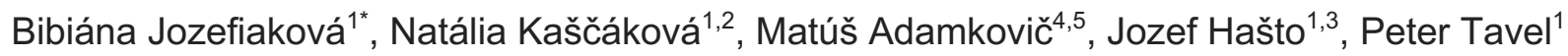 \\ ${ }^{1}$ Olomouc University Social Health Institute, Palacky University Olomouc, Czechia \\ ${ }^{2}$ Psychiatric-Psychotherapeutic Outpatient Clinic, Pro Mente Sana, Slovakia \\ ${ }^{3}$ Department of Social Work, St. Elizabeth College of Health and Social Work, Slovakia \\ ${ }^{4}$ Institute of Psychology, Faculty of Arts, University of Presov, Slovakia \\ ${ }^{5}$ Institute of Social sciences, CSPV Slovak Academy of Sciences, Slovakia \\ *Corresponding author: bibiana.jozefiakova@gmail.com
}

\title{
Data Accessibility
}

The data are available upon request. The analytic code (including covariance matrices) is freely available at https://osf.io/ucd7n/.

\section{Conflict of interest}

None.

\section{Funding}

This work was funded by the Slovak Research and Development Agency [project no. APVV-170418].

\section{Author contribution}

The authors collectively conceived the main idea and the design of the study. BJ wrote the theoretical framework and discussion with input from NK and MA. MA performed statistical analyses. JH, PT and NK supervised the study. All authors have revised the manuscript, made a substantial contribution to this work, and approved it for publication. 


\section{Abstract}

Despite its negative connotation, surviving trauma can result in improvements in some of the domains of one's life. This phenomenon is known as posttraumatic growth and is usually measured using the Posttraumatic Growth Inventory (PTGI). Given the ambiguous results of the existing validation studies, the present study aimed to verify the psychometric properties of the Slovak version of the (PTGI) in a representative sample of Slovak citizens. Although the results suggest that a modified one-factor structure fitted the data best, other issues such as extremely high correlations between the latent factors, related to the PTGl's factor structure were observed. It is likely that the application of the latent variable model does not represent the essence of PTG adequately and the network approach thus appears to be a far more suitable conceptualization of PTG. More detailed information on between-person differences and within-person changes in PTG could help to tailor more effective interventions or preventative programs.

Key words: posttraumatic growth, posttraumatic growth inventory, PTGI, validation, network analysis 


\section{STRUCTURE OF POSTTRAUMATIC GROWTH}

\section{Introduction}

Approximately $70 \%$ of people experience at least one potentially traumatic event in their lifetime (Benjet et al., 2016; Knipscheer et al., 2020). Based on the criteria stated in the DSM-5, trauma is defined as an event in which a person is exposed to actual or imminent death, severe injury, or sexual violence (American Psychiatric Association, 2013). The word trauma is usually perceived negatively and the majority of research on this topic is focused on its negative consequences on mental health. How a person responds to the survival of a traumatic event is conditional on multiple factors (biological, psychosocial, etc.). PTSD is just one of many possible types of reactions (Yehuda et al., 2015), and it develops in about $5 \%$ of people who survived a traumatic event (Atwoli et al., 2015). The prevalence rates stem from a combination of historical circumstances (e.g., war/conflicts, high criminality rates, natural disasters, etc.; see Asnakew et al., 2019) and mental health care infrastructure within individual countries, which determines the practices related to the PTSD diagnosing and treatment.

However, in the last 25 years, research has started to focus also on the positive consequences of surviving a traumatic event. Different terms have been used to describe positive psychological changes after surviving a potentially traumatic event, for instance, positive psychological changes (Yalom \& Lieberman, 1991), stress-related growth (Park et al., 1996), or flourishing (Ryff \& Singer, 1998). The most cited and the most elaborate is the theory of posttraumatic growth (PTG; Tedeschi \& Calhoun, 1996). PTG is defined as a positive change in certain areas of life. The positive psychological change can happen in (at least) one of the following domains: (1) interpersonal relationships, (2) new possibilities, (3) personal strength, (4) spiritual change, and (5) appreciation of life (Tedeschi \& Calhoun, 1996). PTG has been mostly studied in samples of war veterans (Mark et al., 2018), people who survived a natural disaster (García et al., 2015), victims of sexual violence (Bakaityté et al., 2020), oncological patients, or people diagnosed with other serious diseases (Hamama-Raz et al., 2019). Currently, research is focusing on COVID-19 pandemic and its relationship with PTG (Hamam et al., 2021). Working with such specific groups could have narrowed the focus of research on traumatic events to the most extreme ones (e.g., war, natural disaster) despite the fact that people are experiencing a wider range of traumatic events throughout their lives (Brooks et al., 2016). According to Kessler et al. (2017), the three most burdensome traumatic events are sexual violence $(15.1 \%)$, rape $(13.1 \%)$, and unexpected death of a loved one (11.9\%). Mills et al. (2011) found that the most frequently experienced traumatic events are the sudden death of a loved one or witnessing other forms of violence. It is, therefore, important to focus trauma-related research also on the general population (Brooks et 


\section{STRUCTURE OF POSTTRAUMATIC GROWTH}

al., 2016). According to a meta-analysis (Wu et al., 2018), about 53\% of people exposed to some form of a traumatic event (chronically ill people, war veterans, firefighters, rescuers, etc.) consequently experience at least medium posttraumatic growth. A better understanding of posttraumatic growth could significantly improve not only therapeutic work with trauma survivors but also prevention of dysfunctional processing of such events. However, to do so, a valid measure of PTG adapted to different cultural settings is needed first.

With more than 6000 citations, the Posttraumatic Growth Inventory (PTGI) is the most frequently used method for measuring PTG. The items in the original version of the questionnaire were mainly based on the authors' interviews with people who had severe physical difficulties or experienced the death of a loved one (husband/wife) and the questionnaire was validated on a sample of students (Tedeschi \& Calhoun, 1996). There are currently three other versions of the original questionnaire: its shortened form (PTGI-SF; Cann et al., 2010), a version for children (PTGI-C; Cryder et al., 2006), and a version with an expanded spiritual-existential change scale (Tedeschi \& Calhoun, 2017). According to the authors of the original version, the questionnaire consists of five subscales that represent the five PTG domains depicted above. Besides calculating a score for each subscale, a summary score can be elicited (Tedeschi \& Calhoun, 1996). The questionnaire has been validated by several research teams from different countries (e.g., Arandia et al., 2018; Jaarsma et al., 2006; Joseph et al., 2004; Linley et al., 2007; Mack et al., 2015; Osei - Bonsu et al., 2012; Silverstein et al., 2018). The results of these validation studies pointed to the inconsistencies in the factor structure of the PTGI questionnaire. Besides the natural variations caused by language/culture adaptation and sampling, the results may differ due to the use of different statistical procedures to verify the factor structure of the PTGI. In practice, however, either the initial five-factor model or the general one-factor structure (i.e., a simple summary score) is being widely used (Steffens \& Andrykowski, 2015).

Because of (1) the unclear factor structure of the PTGI and (2) the fact that validity of the original version of a measure does not guarantee that its adaptation to other languages will be valid as well (see, e.g., Byrne, 2016), the main aim of this study was to verify the psychometric properties of the Slovak version of the Posttraumatic growth inventory (PTGI) on a representative sample of Slovaks. Additional goals of this study were to examine the invariance of the instrument across gender and to examine its relations with external variables (spirituality and resilience) and also with the type of traumatic or stressful event. Ultimately, the structure of the PTGI was explored using the network analysis. 
STRUCTURE OF POSTTRAUMATIC GROWTH

\section{Method}

\section{Participants and procedure}

Data were collected in April 2019. Based on quota characteristics (gender, age, education, size of the place of residence, and region of residence), a total of 1018 respondents were selected. Quota characteristics were calculated based on the data from the Statistical Office of the Slovak Republic. More descriptive data about the sample are available in Table 1. Using the Life stressor checklist (LSC-R), we identified that $71 \%(\mathrm{~N}=721)$ of people in the sample survived a traumatic or stressful life event. Those participants were then administered the Posttraumatic growth inventory (PTGI). The study was reviewed and approved by the ethics board of the Olomouc University Social Health Institute (OUSHI).

\section{Measures}

\section{Posttraumatic growth inventory (PTGI)}

The posttraumatic growth inventory (PTGI) measures the level of posttraumatic growth in persons who have survived a traumatic event (Tedeschi \& Calhoun, 1996). It consists of 21 items. Each of them falls under one of the five factors: (1) relating to others, (2) new opportunities, (3) personal strength, (4) spiritual change, and (5) understanding of life. The participants are asked to indicate the degree to which they have or have not experienced a particular change using the scale ranging from 0 to 5 . A higher score indicates a higher level of posttraumatic growth. Examples of items: (1) I'm more aware that I can handle difficulties, (2) I'm putting more effort into my relationships, or (3) l've found out how great people are.

\section{Brief resilience scale (BRS)}

Resilience was measured using the Brief resilience scale (BRS; Smith et al., 2008). The BRS consists of six items and measures resilience as the ability to recover from a stressful event. Examples of items: (1) It is difficult for me to go through a stressful situation or (2) I tend to recover quickly from difficult situations. The reliability of the scale was $\omega_{\text {total }}=.87$.

\section{Spiritual well-being scale (SWBS)}

The Spiritual well-being scale (SWBS; Paloutzian and Ellison, 1982) is a self-report questionnaire that measures spiritual and life well-being. The SWBS consists of 20 items, from which either a 
summary score can be calculated or two subscales (religious well - being and existential wellbeing) could be elicited. In this sample, the scale showed high reliability with $\omega_{\text {total }}=.87$.

\section{Functional assessment of chronic illness therapy (FACIT)}

FACIT is a 12-item self-report questionnaire designed to measure spiritual well-being and quality of life for people diagnosed with serious diseases (Peterman et al., 2002). Four items of the questionnaire focus on meaning and purpose of life, four items on inner peace, and four items on faith. The reliability of the whole scale was high with $\omega_{\text {total }}=.88$.

\section{Life stressor checklist (LSC)}

The prevalence of exposure to life stressors was assessed by the Life stressor checklist (Wolfe et al., 1996). The LSC is a 30-item questionnaire; 19 items assess psychological trauma, and nine items focus on stressful life events. Additional questions provide insights into the age of the person at the time of surviving the event and how much this situation affects them in later life (Kaščáková et al., 2018).

Table 1. Demographic characteristics of the sample

\begin{tabular}{lcc}
\hline & $\mathbf{N}$ & $\mathbf{\%}$ \\
\hline Gender & & \\
Male & 496 & 48,7 \\
Female & 522 & 51,3 \\
Age & & \\
$18-24$ y. & 110 & 10,8 \\
$25-34$ y. & 187 & 18,4 \\
$35-44$ y. & 199 & 19,5 \\
$45-54$ y. & 166 & 16,3 \\
$55-64$ y. & 168 & 16,5 \\
65 or more & 188 & 18,5 \\
Living with & & \\
A partner & 671 & 65.9 \\
Alone & 162 & 15,9 \\
Parents & 185 & 18,2 \\
Level of education & & \\
Primary & 137 & 13,5 \\
Secondary vocational & 272 & 26,7 \\
school & 382 & 37,5 \\
High school & 227 & 22,3 \\
University degree & &
\end{tabular}




\section{Data analysis}

In the initial screening, descriptive statistics were calculated, and the data were screened for missings and improbable value. Given the online administration, the particular variables did not contain any missing values or values that were unlikely to occur (e.g., typos) - all the observed values were within the range of the response scales. As such, no observations were considered as outliers and no transformation of data had been applied. The initial screening also included inspection of the correlation matrix of the PTGI items.

Afterwards, the dataset was randomly split into two parts - exploratory $\left(\mathrm{N}_{\mathrm{E}}=360\right)$ and confirmatory $\left(\mathrm{N}_{\mathrm{C}}=361\right)$. A priori analysis of statistical power based on the RMSEA coefficient $\left(\alpha=.05 ; \mathrm{H}_{\mathrm{A}}\right.$ RMSEA = .08; $\mathrm{H}_{0}$ RMSEA = .04) indicated that for a combination of a sample of $\mathrm{N}=360$ and a model with $\mathrm{df}=179$ (the Five-factor model), statistical power to detect model's misspecification converges to $100 \%$. Although both PTGI models were constructed in accordance with the conventions in PTG research (Tedeschi \& Calhoun, 1996; Silverstein et al., 2018), the hereemployed form of cross-validation was preferred as the occurrence of some misspecifications was expected. The exploratory dataset served for addressing these misspecifications (all the potential modifications had to be, first and foremost, theoretically justifiable). The confirmatory dataset was hence used to cross-validate the results and to select the most optimal PTGI structure. Consequent invariance testing (with gender as a potential source of invariance), and examination of convergent validity was performed only for the best-fitting model. When examining convergent validity, external variables (BRS, SWBS, and FACIT) were modelled together in one general model (which, obviously, included also PTGI) and correlations between the latent variables were calculated. From the technical perspective, the models were initially estimated using the WLSMV method, with the items being treated as ordinal. The models were also fitted using the $\mathrm{ML}$ estimator due to the technical problems with fitting the Five-factor model using the WLSMV (see Results), as well as for the purpose of comparison of competing models using the chi-square difference test. The models were considered disconfirmed had the value of chi-square (note, chi-square is the only statistical test of model-data fit in SEM; see Ropovik, 2015) been significant. The potential sources of models' misfit were inspected (factor loadings, covariances between latent factors, residual matrix, and modification indices were checked). Apart from calculating chi-square values, fit of the models was diagnosed using the (scaled) conventional approximate fit indices (AFI), namely, CFI, TLI, RMSEA, and SRMR (see Hu \& Bentler, 1999). Given the nested structure of the tested models (expect the modified One-factor model), a formal chi-square difference test was calculated to determine which of the models fit the data best. To 
STRUCTURE OF POSTTRAUMATIC GROWTH examine the reliability of the respective factors, McDonald's omega (utilizing polychoric correlations) was computed.

With regard to the problematic and hardly interpretable results of the performed CFAs (see Results below), a network analysis was calculated (as an exploratory part of this study) on the whole dataset. The traditional, more or less implicit assumption of a latent variable which causally determines the observed (measured) behaviors is, in fact, only hardly justifiable for the conceptualization of psychological constructs (e.g., Borsboom, 2008). To the contrary, PTG (or, eventually, any other psychological construct; see, e.g., Borsboom \& Cramer, 2013) is composed of a set of indicators that are mutually connected and have an inner structure. As, in respect to the goals of this paper, computing a network of the PTGI items had not been initially intended, the below-presented network has more of a demonstrative (rather than technically rigorous) purpose. The network was estimated using the EBICglasso estimator (the tuning parameters were set to prefer sparser network). Centrality/connectivity parameters, as well as indices of network stability and replicability, were calculated. The analyses were performed in R (R Core Team, 2020), with psych (Revelle, 2020), lavaan (Rosseel, 2012), qgraph (Epskamp et al., 2012), and bootnet (Epskamp et al., 2018) serving as the main packages.

\section{Results}

A correlation matrix, as well as means and standard deviations of the PTGI items, is available in Table 2. 
Table 2. Correlation matrix, means and standard deviations of the PTGI items

\begin{tabular}{|c|c|c|c|c|c|c|c|c|c|c|c|c|c|c|c|c|c|c|c|c|c|}
\hline & 1 & 2 & 3 & 4 & 5 & 6 & 7 & 8 & 9 & 10 & 11 & 12 & 13 & 14 & 15 & 16 & 17 & 18 & 19 & 20 & 21 \\
\hline 1 & - & & & & & & & & & & & & & & & & & & & & \\
\hline 2 & .81 & - & & & & & & & & & & & & & & & & & & & \\
\hline 3 & .70 & .73 & - & & & & & & & & & & & & & & & & & & \\
\hline 4 & .65 & .68 & .76 & - & & & & & & & & & & & & & & & & & \\
\hline 5 & .57 & .61 & .55 & .57 & - & & & & & & & & & & & & & & & & \\
\hline 6 & .61 & .68 & .64 & .68 & .65 & - & & & & & & & & & & & & & & & \\
\hline 7 & .70 & .70 & .76 & .72 & .62 & 67 & - & & & & & & & & & & & & & & \\
\hline 8 & .62 & .67 & .65 & .69 & .63 & .76 & .74 & - & & & & & & & & & & & & & \\
\hline 9 & .60 & .63 & .62 & .68 & .59 & .68 & .68 & .78 & - & & & & & & & & & & & & \\
\hline 10 & .66 & .68 & .65 & .71 & .59 & .71 & .70 & .71 & .72 & - & & & & & & & & & & & \\
\hline 11 & .71 & .74 & .69 & .72 & .61 & .72 & .73 & .72 & .70 & .86 & - & & & & & & & & & & \\
\hline 12 & .68 & .73 & .67 & .71 & .61 & .73 & .70 & .73 & .70 & .82 & .86 & - & & & & & & & & & \\
\hline 13 & .69 & .74 & .65 & .67 & .59 & .68 & .68 & .69 & .67 & .78 & .82 & .81 & - & & & & & & & & \\
\hline 14 & .64 & .66 & .70 & .70 & .56 & .66 & .73 & .69 & .67 & .72 & .75 & .73 & .72 & - & & & & & & & \\
\hline 15 & .63 & .69 & .64 & .64 & .62 & .70 & .64 & .76 & .70 & .73 & .76 & .74 & .76 & .74 & - & & & & & & \\
\hline 16 & .62 & .66 & .64 & .67 & .52 & .66 & .68 & .73 & .73 & .70 & .74 & .72 & .73 & .68 & .73 & - & & & & & \\
\hline 17 & .65 & .69 & .66 & .69 & .56 & .67 & .70 & .70 & .70 & .74 & .76 & .76 & .75 & .74 & .75 & .80 & - & & & & \\
\hline 18 & .47 & .48 & .44 & .45 & .73 & .51 & .48 & .53 & .50 & .46 & .49 & .49 & .51 & .48 & .55 & .45 & .49 & - & & & \\
\hline 19 & .68 & .71 & .65 & .71 & .60 & .67 & .70 & .68 & .67 & .78 & .79 & .77 & .74 & .72 & .72 & .68 & .75 & .57 & - & & \\
\hline 20 & .60 & .63 & .63 & .68 & .57 & .71 & .66 & .72 & .67 & .69 & .73 & .72 & .70 & .70 & .73 & .70 & .73 & .54 & .76 & - & \\
\hline 21 & .64 & .68 & .64 & .66 & .64 & .74 & .68 & .75 & .71 & .75 & .77 & .77 & .75 & .72 & .81 & .74 & .78 & .57 & .77 & .84 & - \\
\hline$M$ & 2.22 & 2.54 & 2.14 & 2.19 & 1.91 & 2.22 & 2.11 & 2.09 & 2.02 & 2.31 & 2.41 & 2.35 & 2.61 & 2.02 & 2.30 & 2.22 & 2.15 & 1.66 & 2.32 & 2.21 & 2.20 \\
\hline $\mathrm{SD}$ & 1.58 & 1.58 & 1.52 & 1.48 & 1.54 & 1.49 & 1.57 & 1.45 & 1.46 & 1.51 & 1.54 & 1.48 & 1.58 & 1.55 & 1.45 & 1.45 & 1.48 & 1.59 & 1.57 & 1.50 & 1.44 \\
\hline
\end{tabular}




\section{STRUCTURE OF POSTTRAUMATIC GROWTH}

\section{Confirmatory factor analyses}

\section{Exploratory dataset}

The original One-factor model $\left(x^{2}(47)=686.96 ; p<.001\right)$, as well as the original Five-factor model $\left(X^{2}(48)=481.55 ; p<.001\right)$ have shown significant deviations from data. The mean value of the factor loadings was very high $-\lambda=.86$ (ranging from .68 - .94) for the One-factor model and $\lambda=.89$ (ranging from .79- .96) for the Five-factor model, respectively. In the One-factor model, there were observed high residual covariances (cov > .10) between the items no.: 1 and 2, 3 and 4, and 5 and 18. Modification indices $(M I>.10)$ suggested to include a covariance term between 14 pairs of the items, with the absolute values of the standardized expected parameter change ranging from 0.25 to 0.78 . After adding a covariance term between the theoretically justifiable pairs of the items (items no. 5 and 18, 20 and 21, 1 and 2, 10 and 11, 11 and 12, 3 and 4 , and 16 and 17), the value of chi-square statistics dropped substantially. The model was, however, still deemed disconfirmed. Five-factor model, despite it has shown much better fit compared to the One-factor model, had flagged problems with the convergence - correlations between the five latent factors were extremely high (mean correlation was .89), and the estimated correlation between the factor 2 and the factor 3 exceeded the value of 1 . Estimation of the model using ML had still produced similar problems with its convergence. Although there were no visible problems with residual covariation in this case, the values of modification indices had been erroneous. Due to high collinearity between the factors, the factors correlating above .8 were merged, creating a model with two latent factors (items no. 5 and 18 loaded on one factor and all the other items loaded on the second factor). Even though the Two-factor model converged, sources of its misspecifications were similar to those observed in the One-factor model. The comparison of the nested model using the likelihood ratio test has shown that, out of the candidate models, the Five-factor model fitted the data best. When compared with the nonnested modified One-factor model (probabilistic model selection), the Five-factor model had shown worse values of information criteria AIC and BIC. Parameters of models' fit are summarized in Table 3. The reliabilities of the respective subscales (both in the One-factor, the Two-factor, and the Five-factor model) were extremely high $\omega_{\text {Total }}=.90-.98$, except for the reliability of the fourth factor in the Five-factor model, which was still very high ( $\left.\omega_{\text {Total }} .86\right)$. 
STRUCTURE OF POSTTRAUMATIC GROWTH

Table 3. Model fit parameters (scaled) for both the exploratory and the confirmatory dataset

\begin{tabular}{lccccccc}
\hline Model & $\mathrm{X}^{2}$ & $\mathrm{df}$ & $\mathrm{p}$ & $\mathrm{CFI}$ & $\mathrm{TLI}$ & $\mathrm{RMSEA}[95 \% \mathrm{Cl}]$ & SRMR \\
\hline Exploratory dataset & & & & & & & \\
One-factor & 686.96 & 47 & $<.001$ & .87 & .99 & $.20[.18, .21]$ & .04 \\
Five-factor & 481.55 & 48 & $<.001$ & .91 & .99 & $.16[.15, .17]$ & .03 \\
Modified One-factor & 381.62 & 50 & $<.001$ & .93 & .99 & $.14[.12, .15]$ & .03 \\
Two-factor & 603.53 & 47 & $<.001$ & .89 & .99 & $.18[.17, .19]$ & .04 \\
Confirmatory dataset & & & & & & & .04 \\
One-factor & 713.25 & 37 & $<.001$ & .87 & .98 & $.23[.21, .24]$ & .05 \\
Five-factor & 438.70 & 37 & $<.001$ & .92 & .99 & $.17[.16, .19]$ & .04 \\
Mod. One-factor & 379.60 & 38 & $<.001$ & .94 & .99 & $.16[.15, .17]$ & .04 \\
Two-factor & 536.81 & 37 & $<.001$ & .91 & .99 & $.19[.18, .21]$ & .04 \\
\hline
\end{tabular}

\section{Confirmatory dataset}

All the models tested in the exploratory dataset were fitted again in the confirmatory dataset. Similar to the previous results, the Five-factor model had had troubles with convergence regardless of the estimation method, as there was extremely high collinearity between the latent factors. Given the fact that the modified One-factor model had fitted the data best, it was used for further analysis of convergent validity and measurement invariance across gender. Models' fit parameters for all the models are presented in Table 3.

Assuming potential gender differences in the PTGI structure, measurement invariance was calculated on the modified One-factor model. The results indicate that the PTGI structure is invariant across gender in term of metric $\left(\Delta x^{2}=20.92, \Delta d f=20, p=.402\right)$ and scalar $\left(\Delta x^{2}=28.34\right.$, $\Delta d f=20, p=.102)$ invariance. Significant non-invariance was observed in terms of latent means of PTG $\left(\Delta x^{2}=10.12, \Delta d f=1, p=.001\right)$, with women scoring 0.35 SD (converted to the PTGI scale $=9.1$ points) higher than men. 
The modified One-factor model was then correlated with the BRS ( $r=-.05)$, SWBS $(r=.29)$, and FACIT ( $r=.32$ ) within one general model. The reported coefficients thus represent correlations between the latent variables. It is worth noting that the item no. 18 has shown very high crossloadings on both SWBS and FACIT. For descriptive purposes, the One-factor model was correlated with the Life Stressor Checklist (LSC-R), which serves as a screening for stressful (traumatic) experiences. The results of LSC-R were then divided into five scores: (1) interpersonal

violence, (2) indirect trauma, (3) traumatic events experienced before 16 years old, (4) summary score of traumatic exposure, and (5) other forms of trauma (see Schumacher et al., 2010; Kaščáková et al., 2018). The observed correlations between the PTGI score and the abovedescribed scores were $r=.10, r=.22, r=.15, r=.22$, and $r=.23$ for interpersonal violence, indirect trauma, traumatic events experienced before 16 years old, summary score of traumatic exposure, and other forms of trauma, respectively.

\section{PTG as a network}

The performed analyses point out to five main facts: (1) there was a strong correlation (average $r$ = .68) between the PTGI items; (2) all the PTGI items loaded very well on the latent factor/factors; (3) if PTGI was modelled with more than one latent factor, there were extremely high correlations between the factors; (4) the modified One-factor model fitted the data best; (5) none of the PTGI items was problematic per se. The combination of these facts suggests that it is next to impossible to find an optimal factor structure of PTGI (note: this assumes that all the PTGI items are important for the operationalization of PTG - in other words, the items in PTGI capture all the theoretically relevant aspects of $\mathrm{PTG}$ ). It is, therefore, possible that the inability to find the optimal factor structure stems from the way PTG is statistically modelled in the validation studies, including this one. Applying reflective latent models to psychological constructs rests on several assumptions which are usually not tenable. For example, in reality, it is unlikely that there exists an underlying latent variable (or, say, five latent variables) representing PTG that causes its observable indicators. Rather on the contrary, mutual relationships between the specific behavioral aspects (indicators) and their dynamics cause a system which is conventionally labelled as PTG. This radical shift in the perspective and understanding of a phenomenon (from latent models to network approach) subsequently changes the nature of the research questions being asked, for instance: (1) how are the indicators of PTG related? (2) Which PTG indicators play a core, or on the other hand, a peripheral role in the system? To provide an insight into these emerging questions, a network consisting of PTG indicators had been estimated. A visualization of this (conservative - small edges were shrunk to zero) network is presented in Figure 1a. 
Figure 1. A network of PTG indicators and their centrality indices

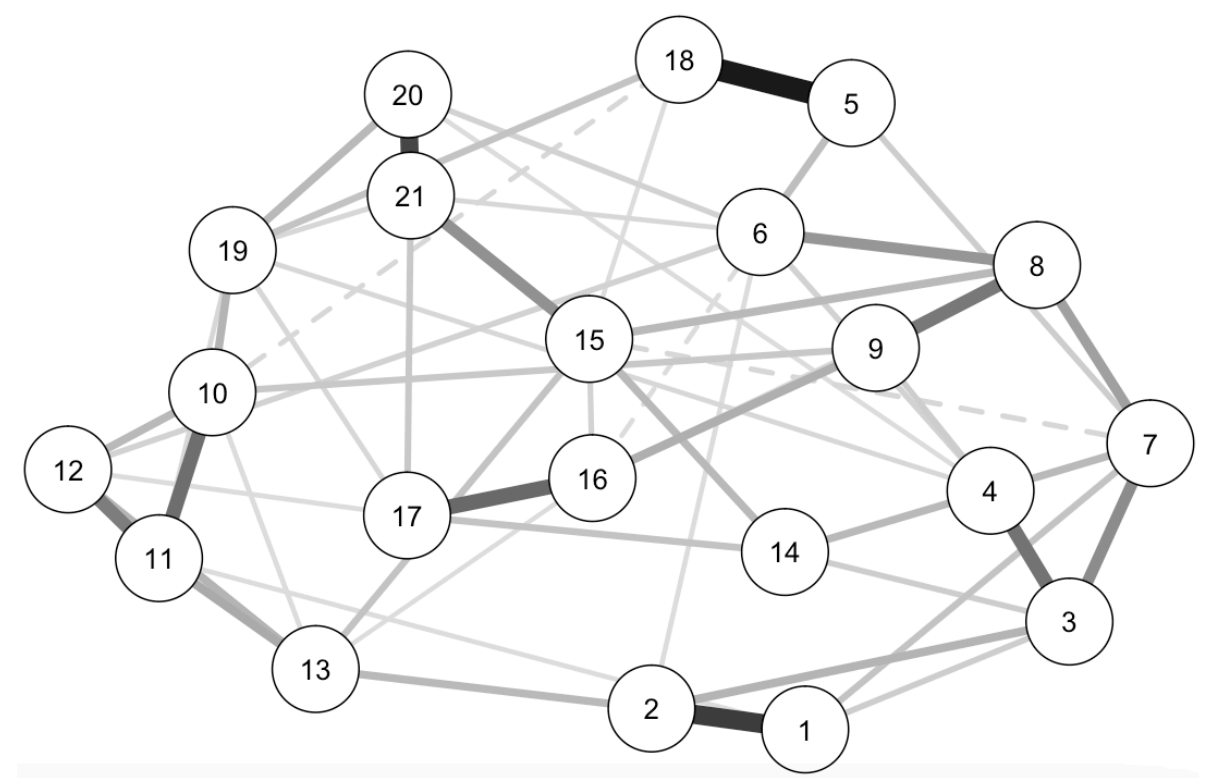

Figure 1a. A visualization of a regularized network of the PTG indicators. Thicker edges depict stronger relationships. Dashed edges indicate negative relationships. The numbers in the nodes correspond with the numbers of the PTGI items.
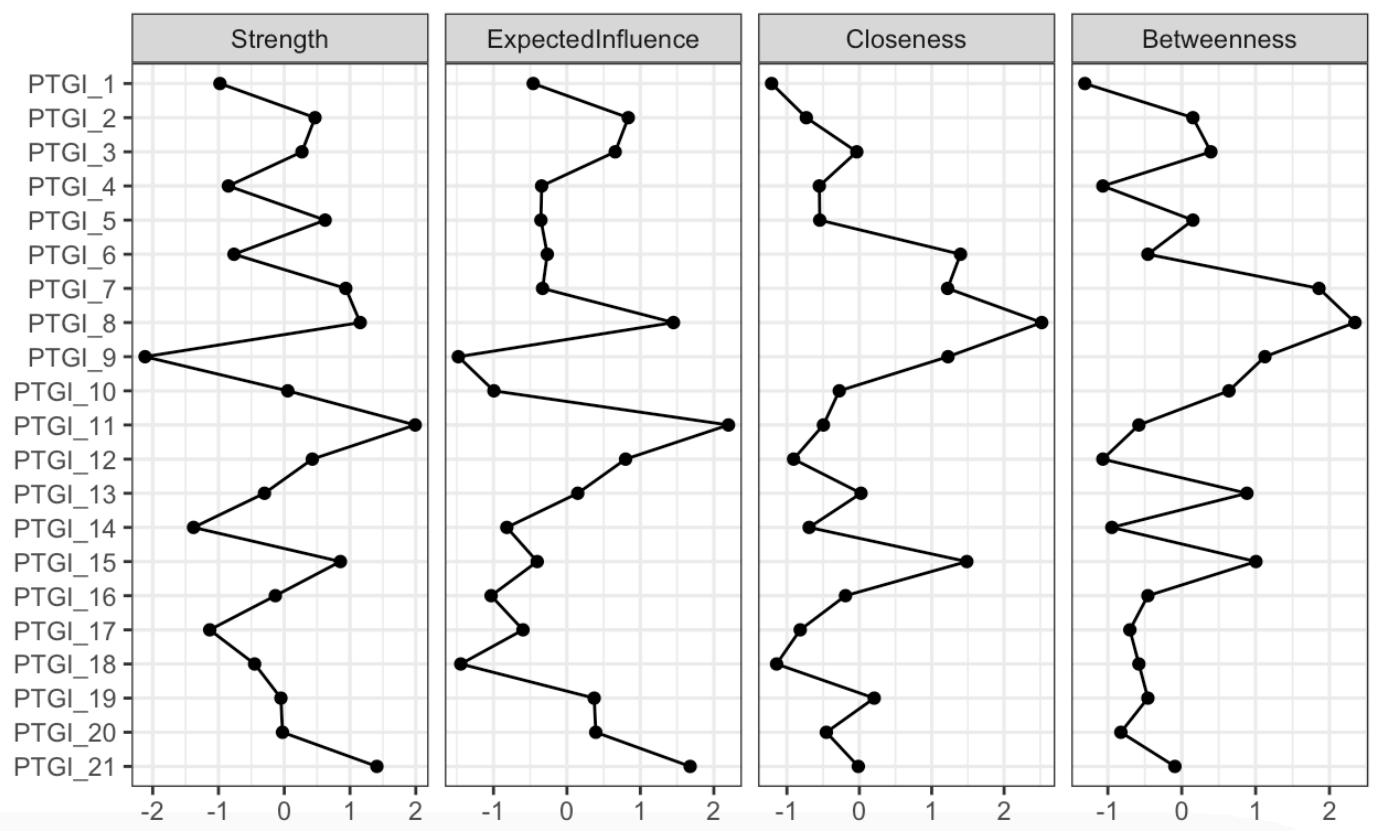

Figure $1 \mathrm{~b}$. Centrality and connectivity indices of the PTG indicators.

As can be seen from Figure $1 \mathrm{~b}$, the highest degree of centrality (strength of a direct association between an indicator and other indicators; strength and expected influence) were found for the 


\section{STRUCTURE OF POSTTRAUMATIC GROWTH}

items no. 11,21 , and 8 . The highest connectivity, in terms of how strongly a node is indirectly connected with other nodes (closeness), was found for the items no. 8, 6, 7, 9, and 15, while the items no. 7 and 8 were the most important for connecting other nodes (betweenness).

The estimated network is relatively robust. Bootstrapped estimates suggest that the abovedescribed parameters are rather stable although the stability is not ideal (for all the parameters, the point estimate of average correlation with the original sample is above $r=.50$ even when $50 \%$ of cases are dropped; see https://osf.io/ucd7n/). The presented network is also fairly replicable. Estimates obtained from replicating and simulating the network highly correlate $(r \approx .70$ and higher) with the original values (except for Jaccard index) even when 250 cases are sampled. Altogether, the network performs well enough to make some initial inferences, nonetheless, future replication studies are very much needed.

\section{Discussion}

The aim of this study was to verify the factor structure of the Slovak version of the Posttraumatic growth inventory (PTGI) on a representative sample of the Slovak population. The analyses revealed strong correlations between the PTGI items, and also extremely strong correlations between the latent factors (had the PTG model included more than one latent factor). The results suggested that a modified one-factor model overperformed the competing models. While the structure of the one-factor model was invariant across gender, a difference in latent means was observed (women scored higher compared to men). The questionnaire is thus applicable to both men and women (Mordeno et al., 2015). The convergent validity of the modified One-factor modified model of the PTGI was examined by correlating the factor with external criteria (FACIT, SWBS, BRS). A weak to moderate positive relationship with spirituality and a weak to a moderate negative relationship with resilience were observed. Similar findings were found in other studies (Danhauer et al., 2013; Paredes \& Pereira, 2017; Shaw et al., 2005).

Although the modified One-factor model fitted the data best, altogether, the observed results (e.g., even the best-fitting model significantly deviated from the data; very high correlations between the items; extremely high correlations between the PTGI subscales if the model consists of more than one latent factor), suggest that the main issue might lie elsewhere. Given the observed results, but also the conceptual basis for measuring psychological constructs, applying the latent variable model (a latent factor is a single cause of the observed/reported indicators) to PTG might 


\section{STRUCTURE OF POSTTRAUMATIC GROWTH}

not be appropriate. Instead, a network approach in which PTG is regarded as a set of mutually interacting indicators that form a structure consensually labelled as PTG is a more appropriate representation (for a similar argumentation in PTSD research see Armour et al., 2017)

The existing evidence (e.g., Silverstein et al., 2018), as well as the present results (high correlations between latent factors), suggest that although posttraumatic growth as a construct can be observed in different domains of life (Tedeschi \& Calhoun, 1996), it is probably the same variable. Dividing the PTGI into factors could potentially help to better capture the nature of this phenomenon from the theoretical perspective. The empirical evidence, however, suggests that this distinction is rather didactical. If a researcher aims to study PTG, a reduction in the number of administered items (e.g., administering a short form of the PTGI, see Cann et al., 2010; Lamela et al., 2014) could save their resources as well as the participants' time and effort, subsequently leading to a higher quality of the obtained data. Therefore, if an item is not essential with respect to the constitutive definition of PTG, it might be worth considering to remove it from the measure. In other words, it might be useful to take a step back and look at the constitutive definition of PTG and utilize the corresponding operationalizations in the questionnaire.

Shifting the perspective from a latent variable model to the more structured network approach would allow detecting the central/peripheral indicators, consequently leading to better-tailored interventions/facilitation strategies. For dynamic systems, suggesting interventions solely from cross-sectional data could be tricky (see Henry et al., 2020) and more longitudinal research and studies using experience sampling will be needed. Based on the performed calculations, the items no. $6,7,8,9,11,15$, and 21 appear to be the most central ones. These items correspond to the first (relating to others) and the second (new possibilities) factor from the original five-factor structure. Based on this, we can consider that the most important in posttraumatic growth could be the quality of relationships and the social environment a person has, how they can communicate their difficulties and how a view of life and confidence in their own coping skills will change. A network structure of PTG was already examined by Bellet et al. (2018). The authors, however, used the short form of the PTGI (PTGI-SF) and were primarily focusing on the cooccurrence of PTG and complicated grief. According to their findings, the core indicators (based on the expected influence measure) of PTG were the items no. 6 and 9 (these correspond to the items no. 7 and 19 in the original, full version of the PTGI questionnaire) These items fall under the new possibilities (II) and personal strength (III) factors. Contrary to the present analysis, the authors did not find sufficient evidence for depicting the relating to others (I) factor as a core aspect of PTG. The authors highlight the importance of the ability to imagine a new way forward 


\section{STRUCTURE OF POSTTRAUMATIC GROWTH}

and, at the same time, their results suggest that greater personal strength might be more important for PTG than relationships with others. The least influential items appeared to be the items no. 1 ("My priorities about what is important in life") and 2 ("An appreciation for the value of my life"), which partially corresponds with the findings from the presented analysis.

Based on the results of invariance testing, women had a higher average score of PTG, which may be related to their higher emotionality and openness to communicate about their own experiences (First et al., 2017). Another possible explanation is that women cope with the situation using more deliberative and reflective rumination, which might lead to higher posttraumatic growth (Vishnevsky et al., 2010). In general, emotion-focused coping strategies (positive reaction, acceptance, denial) are positively related to PTG (Butler et al., 2005; Helgeson et al., 2006; Prati \& Pientrantoni, 2009).

Positive moderate correlations were observed between spirituality, religiosity and posttraumatic growth. This result is in line with the theory of posttraumatic growth process as described by Tedeschi and Calhoun (1996; spiritual change is one of the main aspects of PTG) and is also supported by other studies (e.g., Prati \& Pientrantoni, 2009). Furthermore, weak positive correlations were observed between PTG and the different types of stressful events as measured by the Life Stressor Checklist. The type of traumatic event is related to posttraumatic growth only marginally and it appears that subjective evaluation of the traumatic situation (i.e., subjectively perceived burden and stress) play a more important role compared to its objective attributes (Karanci et al., 2012). A weak negative correlation was also observed between PTG and resilience. At the first glance, it may seem that these concepts are very similar, even synonymic, and such finding is counterintuitive. The evidence on this topic is, however, inconsistent. While some authors found a positive relationship between these two variables (Bensimon, 2012), others detected a negative relationship (Levine et al., 2009). The inverse correlation between resilience and PTG could be explained by the fact that a more resilient person may not cognitively evaluate (cognitive processing plays a key role in PTG development; Tedeschi \& Calhoun, 1996) a traumatic event as sufficiently threatening or disruptive and, as such, PTG may not develop (Levine et al., 2009). The results may also vary due to a very pragmatic reason - the fact that different operationalizations of a construct could lead to different findings (see, e.g., Adamkovič et al., 2020; Bensimon, 2012). 


\section{STRUCTURE OF POSTTRAUMATIC GROWTH}

\section{Limits and perspectives of further research}

The present study has several limitations. The first one regards the research sample. As the data comes from a representative sample of the adult Slovak population, the generalizability of the results to other cultural settings might be, obviously, limited. Second, the study was not focused on discussing the theoretical justification of the constitutive definition of PTG nor it was focused on qualitative analysis of items operationalization. Third, even though the study presented also a network analysis of PTG, it is important to acknowledge that this was not the original purpose of the study (the main aim of the study was to verify the psychometric properties and the factor structure of the PTGI) and, as such, the here-presented network has rather a demonstrative character. To learn more about the structure of PTG from a network perspective, more exclusive research on this topic would be needed. Ideally, such research would combine cross-sectional, longitudinal, and experiential sampling design, while putting sufficient effort into having the study designs reasonably powered. The combination of between-person differences and within-person changes could help to determine which aspects of PTG are more efficient to address by potential interventions or prevention programs. Having a succinct PTG measure with a clear structure which produces a valid score across different cultures is, therefore, a necessity.

\section{Conclusion}

The PTGI (Tedeschi \& Calhoun, 1996) is the most commonly used method for measuring posttraumatic growth. The questionnaire was validated on different samples (e.g., general population, students population, people diagnosed with serious diseases) and cultural settings. Its factor structure and psychometric properties vary across these validations. One of the main reasons for this ambiguity might be the fact that the application of the latent variable model does not represent the essence of PTG adequately and the network approach thus appears to be a more suitable conceptualization. Shifting the perspective could significantly help to grasp the construct and consequently to target more effective interventions. A better understanding of PTG could significantly help both prevention and intervention in psychotraumatology. Having a succinct PTG measure with a clear structure which produces a valid score across different cultures would substantially help in pursuing this goal. A collaborative and transparent approach could greatly advance this ambition. 


\section{STRUCTURE OF POSTTRAUMATIC GROWTH}

\section{References}

Adamkovič, M., Martončík, M., Lačný, M., \& Kačmárová, M. (2020, preprint). Poverty in behavioural research: Different operationalizations, different results. doi:10.31234/osf.io/4ek5y

American Psychiatric Association. (2013). Diagnostic and statistical manual of mental disorders (5th ed.). Washington, DC, American Psychiatric Association

Arandia, A. M. H., Mordeno, I. G., \& Nalipay, M. J. N. (2018). Assessing the latent structure of posttraumatic growth and its relationship with cognitive processing of trauma among Filipino women victims of intimate partner abuse. Journal of Interpersonal Violence, 33(18), 28492866.doi:10.1177/0886260516632354

Armour, C., Fried, E. I., \& Olff, M. (2017). PTSD symptomics: network analyses in the field of psychotraumatology. European Journal of Psychotraumatology, 8(sup3), 1398003. doi:10.1080/20008198.2017.1398003

Asnakew, S., Shumet, S., Ginbare, W., Legas, G., \& Haile, K. (2019). Prevalence of posttraumatic stress disorder and associated factors among Koshe landslide survivors, Addis Ababa, Ethiopia: a community-based, cross-sectional study. BMJ Open, 9(6), e028550. doi:10.1136/bmjopen2018-028550

Atwoli, L., Stein, D. J., Koenen, K. C., \& McLaughlin, K. A. (2015). Epidemiology of posttraumatic stress disorder: Prevalence, correlates and consequences. Current Opinion in Psychiatry, 28(4), 307-311. doi: 10.1097/YCO.0000000000000167

Bakaityté, A., Kaniušonytè, G., Truskauskaitè-Kunevičienè, I., \& Žukauskienè, R. (2020). Longitudinal investigation of posttraumatic growth in female survivors of intimate partner violence: The role of event centrality and identity exploration. Journal of Interpersonal Violence. doi: $10.1177 / 0886260520920864$

Bellet, B.W., Jones, P.J., Neimeyer, R.A., \& McNally, R.J. (2018). Bereavevement Outcomes as Causal Systems: A Network Analysis of the Co-Occurrence of Complicated Grief and Posstraumatic Growth. Clinical Psychological Science, 6(6), 797-809. doi: $10.1177 / 2167702618777454$ 
Benjet, C., Bromet, E., Karam, E. G., Kessler, R. C., McLaughlin, K. A., Ruscio, A. M., Shahly, V., Stein, D. J., Petukhova, M., Hill, E., Alonso, J., Atwoli, L., Bunting, B., Bruffaerts, R., Caldas-deAlmeida, J. M., de Girolamo, G., Florescu, S., Gureje, O., Huang, Y., ... Koenen, K. C. (2016). The epidemiology of traumatic event exposure worldwide: results from the World Mental Health Survey Consortium. Psychological Medicine, 46(2), 327-343. doi: 10.1017/S0033291715001981

Bensimon, M. (2012). Elaboration on the association between trauma, PTSD and posttraumatic growth: The role of trait resilience. Personality and Individual Differences, 52(7), 782-787. doi: 10.1016/j.paid.2012.01.011

Borsboom, D. (2008). Psychometric perspectives on diagnostic systems. Journal of Clinical Psychology, 64(9), 1089-1108. doi:10.1002/jclp.20503

Borsboom, D., \& Cramer, A. O. J. (2013). Network analysis: an integrative approach to the structure of psychopathology. Annual Review of Clinical Psychology, 9(1), 91-121. doi:10.1146/annurev-clinpsy-050212-185608

Brooks, M., Lowe, M., Graham-Kevan, N., \& Robinson, S. (2016). Posttraumatic growth in students, crime survivors and trauma workers exposed to adversity. Personality and Individual Differences, 98, 199-207. doi: 10.1016/j.paid.2016.04.051

Butler, L. D., Blasey, C. M., Garlan, R. W., McCaslin, S. E., Azarow, J., Chen, X.-H., Desjardins, J. C., DiMiceli, S., Seagraves, D. A., Hastings, T. A., Kraemer, H. C., \& Spiegel, D. (2005). Posttraumatic growth following the terrorist attacks of September 11, 2001: Cognitive, coping, and trauma symptom predictors in an internet convenience sample. Traumatology, 11(4), 247267. doi: $10.1177 / 153476560501100405$

Byrne, B. M. (2016). Adaptation of assessment scales in cross-national research: Issues, guidelines, and caveats. International Perspectives in Psychology: Research, Practice, Consultation, 5(1), 51-65. doi:10.1037/ipp0000042

Cann, A., Calhoun, L. G., Tedeschi, R. G., Taku, K., Vishnevsky, T., Triplett, K. N., \& Danhauer, S. C. (2010). A short form of the Posttraumatic Growth Inventory. Anxiety, Stress, and Coping, 23(2), 127-137. doi: 10.1080/10615800903094273 


\section{STRUCTURE OF POSTTRAUMATIC GROWTH}

Cryder, C. H., Kilmer, R. P., Tedeschi, R. G., \& Calhoun, L. G. (2006). An exploratory study of posttraumatic growth in children following a natural disaster. American Journal of Orthopsychiatry, 76(1), 65-69. doi: 10.1037/0002-9432.76.1.65ň

Danhauer, S. C., Case, L. D., Tedeschi, R., Russell, G., Vishnevsky, T., Triplett, K., ... Avis, N. E. (2013). Predictors of posttraumatic growth in women with breast cancer. Psycho-Oncology, 22(12), 2676-2683. doi:10.1002/pon.3298

Epskamp, S., Borsboom, D., \& Fried, E. I. (2018). Estimating psychological networks and their accuracy: A tutorial paper. Behavior Research Methods, 50(1), 195-212. doi:10.3758/s13428017-0862-1

Epskamp, S., Cramer, A. O. J., Waldorp, L. J., Schmittmann, V. D., \& Borsboom, D. (2012). qgraph: Network Visualizations of Relationships in Psychometric Data. Journal of Statistical Software, 48(4). doi:10.18637/jss.v048.i04

Ferry, F., Bunting, B., Murphy, S., O'Neill, S., Stein, D., \& Koenen, K. (2013). Traumatic events and their relative PTSD burden in Northern Ireland: a consideration of the impact of the "Troubles." Social Psychiatry and Psychiatric Epidemiology, 49(3), 435-446. doi:10.1007/s00127-013-07570

First, J., First, N., Stevens, J., Mieseler, V., \& Houston, J. B. (2017). Post-traumatic growth 2.5 years after the 2011 Joplin, Missouri tornado. Journal of Family Social Work, 21(1), 5-21. doi:10.1080/10522158.2017.1402529

García, F. E., Cova, F., Rincón, P., \& Vázquez, C. (2015). Trauma or growth after a natural disaster? The mediating role of rumination processes. European Journal of Psychotraumatology, 6(1). doi:10.3402/ejpt.v6.26557

Hamam, A. A., Milo, S., Mor, I., Shaked, E., Eliav, A. S., \& Lahav, Y. (2021). Peritraumatic reactions during the COVID-19 pandemic - The contribution of posttraumatic growth attributed to prior trauma. Journal of Psychiatric Research, 132, 23-31. doi:10.1016/j.jpsychires.2020.09.029

Hamama-Raz, Y., Pat-Horenczyk, R., Roziner, I., Perry, S., \& Stemmer, S. M. (2019). Can posttraumatic growth after breast cancer promote positive coping? A cross-lagged study. PsychoOncology, 28(4), 767-774. doi:10.1002/pon.5017 


\section{STRUCTURE OF POSTTRAUMATIC GROWTH}

Helgeson, V. S., Reynolds, K. A., \& Tomich, P. L. (2006). A meta-analytic review of benefit finding and growth. Journal of Consulting and Clinical Psychology, 74(5), 797-816. doi:10.1037/0022006X.74.5.797

Henry, T. R., Robinaugh, D., \& Fried, E. I. (2020). On the control of psychological networks. In PsyArXiv. doi:10.31234/osf.io/7vpz2

Hu, L.-t., \& Bentler, P. M. (1999). Cutoff criteria for fit indexes in covariance structure analysis: Conventional criteria versus new alternatives. Structural Equation Modeling, 6(1), 1-55. doi: $10.1080 / 10705519909540118$

Jaarsma, T. A., Pool, G., Sanderman, R., \& Ranchor, A. V. (2006). Psychometric properties of the Dutch version of the posttraumatic growth inventory among cancer patients. PsychoOncology, 15(10), 911-920. doi: 10.1002/pon.1026

Joseph, S., Alex Linley, P., \& Harris, G. J. (2004). Understanding positive change following trauma and adversity: Structural clarification. Journal of Loss \& Trauma, 10(1), 83-96. doi:10.1080/15325020490890741

Karanci, A. N., Işıklı, S., Aker, A. T., Gül, E. İ., Erkan, B. B., Ozkol, H., \& Güzel, H. Y. (2012). Personality, posttraumatic stress and trauma type: factors contributing to posttraumatic growth and its domains in a Turkish community sample. European Journal of Psychotraumatology, 3(1). doi:10.3402/ejpt.v3i0.17303

Kaščáková, N. et al. (2018). The Life Stressor Checklist (LSC-R): The occurrence of stressors in adult Czech population and the association with health. Československá Psychologie, 62(1): 8099

Kessler, R. C., Aguilar-Gaxiola, S., Alonso, J., Benjet, C., Bromet, E. J., Cardoso, G., Degenhardt, L., de Girolamo, G., Dinolova, R. V., Ferry, F., Florescu, S., Gureje, O., Haro, J. M., Huang, Y., Karam, E. G., Kawakami, N., Lee, S., Lepine, J.-P., Levinson, D., ... Koenen, K. C. (2017). Trauma and PTSD in the WHO World Mental Health surveys. European Journal of Psychotraumatology, 8(sup5). doi:10.1080/20008198.2017.1353383

Knipscheer, J., Sleijpen, M., Frank, L., de Graaf, R., Kleber, R., Ten Have, M., \& Dückers, M. (2020). Prevalence of potentially traumatic events, other life events and subsequent reactions indicative for posttraumatic stress disorder in the Netherlands: A general population study based 


\section{STRUCTURE OF POSTTRAUMATIC GROWTH}

on the Trauma Screening Questionnaire. International Journal of Environmental Research and Public Health, 17(5), 1725. doi:10.3390/ijerph17051725

Lamela, D., Figueiredo, B., Bastos, A., \& Martins, H. (2014). Psychometric properties of the Portuguese version of the Posttraumatic Growth Inventory Short Form among divorced adults. European Journal of Psychological Assessment: Official Organ of the European Association of Psychological Assessment, 30(1), 3-14. doi:10.1027/1015-5759/a000161

Levine, S. Z., Laufer, A., Stein, E., Hamama-Raz, Y., \& Solomon, Z. (2009). Examining the relationship between resilience and posttraumatic growth. Journal of Traumatic Stress, 22(4), 282-286. doi:10.1002/jts.20409

Linley, A. P., Andrews, L., \& Joseph, S. (2007). Confirmatory factor analysis of the posttraumatic growth inventory. Journal of Loss \& Trauma, 12(4), 321-332. doi: 10.1080/15325020601162823

Mack, J., Herrberg, M., Hetzel, A., Wallesch, C. W., Bengel, J., Schulz, M., ... Schönberger, M. (2015). The factorial and discriminant validity of the German version of the Post-traumatic Growth Inventory in stroke patients. Neuropsychological Rehabilitation, 25(2), 216-232. doi:10.1080/09602011.2014.918885

Mark, K. M., Stevelink, S. A. M., Choi, J., \& Fear, N. T. (2018). Post-traumatic growth in the military: a systematic review. Occupational and Environmental Medicine, 75(12), 904-915. doi:10.1136/oemed-2018-105166

Mills, K. L., McFarlane, A. C., Slade, T., Creamer, M., Silove, D., Teesson, M., \& Bryant, R. (2011). Assessing the prevalence of trauma exposure in epidemiological surveys. The Australian and New Zealand Journal of Psychiatry, 45(5), 407-415. doi:10.3109/00048674.2010.543654

Mordeno, I.G., Nalipay, M.J.N., Alfonso, M. K. S., \& Cue, M. P. (2015). Examining the Latent Structure of Posttraumatic Growth Between Male and Female Survivors in the Immediate Aftermath of a Flash Flood Disaster. Current Psychology, 35/4), 587-594. doi:10.1007/s12144015-9325-y

Osei-Bonsu, P. E., Weaver, T. L., Eisen, S. V., \& Vander Wal, J. S. (2012). Posttraumatic Growth Inventory: Factor Structure in the Context of DSM-IV Traumatic Events. ISRN Psychiatry, 2012, 1-9. doi:10.5402/2012/937582 


\section{STRUCTURE OF POSTTRAUMATIC GROWTH}

Paloutzian, R. F., \& Ellison, C. W. (1982). Loneliness, Spiritual Well-Being and the Quality of Life. In L. A. Peplau, \& D. Perlman (Eds.), Loneliness: A Sourcebook of Current Theory, Research and Therapy (pp. 224-236). New York: John Wiley \& Sons.

Paredes, A. C., \& Pereira, M. G. (2017). Spirituality, Distress and Posttraumatic Growth in Breast Cancer Patients. Journal of Religion and Health. doi:10.1007/s10943-017-0452-7

Park, C. L., Cohen, L. H., \& Murch, R. L. (1996). Assessment and Prediction of Stress-Related Growth. Journal of Personality, 64(1), 71-105. doi:10.1111/j.1467-6494.1996.tb00815.x

Peterman, A. H., Fitchett, G., Brady, M. J., Hernandez, L., \& Cella, D. (2002). Measuring spiritual well-being in people with cancer: the functional assessment of chronic illness therapy--Spiritual Well-being Scale (FACIT-Sp). Annals of Behavioral Medicine: A Publication of the Society of Behavioral Medicine, 24(1), 49-58. doi:10.1207/S15324796ABM2401_06

Prati, G., \& Pietrantoni, L. (2009). Optimism, social support, and coping strategies as factors contributing to posttraumatic growth: A meta-analysis. Journal of Loss \& Trauma, 14(5), 364-388 doi:10.1080/15325020902724271

R Core Team. (2020). R: A language and environment for statistical computing. R Foundation for Statistical Computing, Vienna, Austria. Available at http://www.R-project.org/

Revelle W (2020). psych: Procedures for Psychological, Psychometric, and Personality Research. Northwestern University, Evanston, Illinois. R package version 2.0.9.

Ropovik, I. (2015). A cautionary note on testing latent variable models. Frontiers in Psychology, 6. doi:10.3389/fpsyg.2015.01715

Rosseel, Y. (2012). lavaan: AnRPackage for Structural Equation Modeling. Journal of Statistical Software, 48(2). doi:10.18637/jss.v048.i02

Ryff, C. D., \& Singer, B. (1998). The role of purpose in life and personal growth in positive human health. In P. T. P. Wong \& P. S. Fry (Eds.), The human quest for meaning: A handbook of psychological research and clinical applications (pp. 213-235). Mahwah, NJ: Lawrence Erlbaum Associates, Inc 


\section{STRUCTURE OF POSTTRAUMATIC GROWTH}

Shaw, A., Joseph, S., \& Linley, P. A. (2005). Religion, spirituality, and posttraumatic growth: a systematic review. Mental Health, Religion \& Culture, 8(1), 1-11. doi:10.1080/1367467032000157981

Schumacher, A. M., Jaramillo, D., Uribe, T., De Pheils, P. B., Holzemer, W., Taylor, D., Tiwari, A., Canaval, G. E., Flores, M. E. M., Belknap, R. A., \& Humphreys, J. C. (2010). The relationship of two types of trauma exposure to current physical and psychological symptom distress in a community sample of Colombian women: why interpersonal violence deserves more attention. Health Care for Women International, 31(10), 946-961.doi: 10.1080/07399332.2010.503290

Silverstein, M. W., Witte, T. K., Lee, D. J., Kramer, L. B., \& Weathers, F. W. (2018). Dimensions of growth? Examining the distinctiveness of the five factors of the Posttraumatic Growth Inventory: Factors of the posttraumatic growth inventory. Journal of Traumatic Stress, 31(3), 448-453. doi:10.1002/jts.22298

Smith, B. W., Dalen, J., Wiggins, K., Tooley, E., Christopher, P., \& Bernard, J. (2008). The brief resilience scale: assessing the ability to bounce back. International Journal of Behavioral Medicine, 15(3), 194-200. doi:10.1080/10705500802222972

Steffens, R. F., \& Andrykowski, M. A. (2015). Posttraumatic growth inventory: Overview. In Comprehensive Guide to Post-Traumatic Stress Disorder (pp. 1-14). Springer International Publishing. doi:10.1007/978-3-319-08613-2_12-1

Tedeschi, R. G., \& Calhoun, L. G. (1996). The Posttraumatic Growth Inventory: Measuring the positive legacy of trauma. Journal of Traumatic Stress, 9(3), 455- 472. doi:10.1002/jts.2490090305

Tedeschi, R. G., Cann, A., Taku, K., Senol-Durak, E., \& Calhoun, L. G. (2017). The posttraumatic growth inventory: A revision integrating existential and spiritual change: Posttraumatic growth inventory and spiritual change. Journal of Traumatic Stress, 30(1), 11-18. doi:10.1002/jts.22155

Vishnevsky, T., Cann, A., Calhoun, L. G., Tedeschi, R. G., \& Demakis, G. J. (2010). Gender Differences in Self-Reported Posttraumatic Growth: A Meta-Analysis. Psychology of Women Quarterly, 34(1), 110-120. doi:10.1111/j.1471-6402.2009.01546.x 
STRUCTURE OF POSTTRAUMATIC GROWTH

Wolfe, J., Kimerling, R., Brown, P. J., Chrestman, K. R., Levin, K. (1996): Psychometric review of the Life Stressor Checklist-Revised. Measurement of Stress, Trauma, and Adaptation, 198-201.

Yalom, I. D., \& Lieberman, M. A. (1991). Bereavement and Heightened Existential Awareness. Psychiatry, 54(4), 334-345. doi:10.1080/00332747.1991.11024563

Yehuda, R., Hoge, C. W., McFarlane, A. C., Vermetten, E., Lanius, R. A., Nievergelt, C. M., ... Hyman, S. E. (2015). Post-traumatic stress disorder. Nature Reviews Disease Primers, 1(1). doi:10.1038/nrdp.2015.57 